\title{
Density Driven Image Coding for Tumor Detection in mri Image
}

\author{
Somashekhar Swamy ${ }^{1}$, P.K.Kulkarni ${ }^{2}$ \\ ${ }^{1}$ Research scholar,V.T.U. Belagavi, Karnataka, India., \\ ${ }^{2}$ Professor \& H.O.D.(E\&EE), P.D.A.College of Engineering. Kalburgi, Karnataka, India.
}

\begin{abstract}
The significant of multi spectral band resolution is explored towards selection of feature coefficients based on its energy density. Toward the feature representiaon in transformed domain, multi wavelet transformations were used for finer spectral representation. However, due to a large feature count these features are not optimal under low resource computing system. In the recognition units, running with low resources a new coding approach of feature selection, considering the band spectral density is developed. The effective selection of feature element, based on its spectral density achieve two objective of pattern recognition, the feature coefficient representiaon is minimized, hence leading to lower resource requirement, and dominant feature representation, resulting in higher retrieval performance.
\end{abstract}

Index Term: Spectral features, spectral density feature selection, pattern recognition, retrieval accuracy.

\section{Introduction}

The process of pattern recognition is now emerging at a very rapid rate, with its applications, been diversified from basic school level learning to high precision applications, such as medical applications, military applications etc. wherein all these applications, the basic motivation is to retrieve information for observed patterns from a remote server, the process of retrievation and the descriptive features describing the observation plays an important role. Various approaches were observed in past literature to improvise the retrieval accuracy by introducing new process of recognition methods, or by proposing new feature representations, to achieve higher recognition.Towards such approaches in [1] for the purpose of efficiently and effectively retrieving the desired images from a large image database, the development of a user-friendly image retrieval system is developed. In [2] an approach for feature extraction using wavelet transforms using its property of multilevel decomposition in pattern recognition application is proposed. The multilevel decomposition property of discrete wavelet transform provides information of an image at different resolutions. Towards the applicability of DWT based feature representation in [3] an iris feature extraction method based on wavelet-based contourlet transform (WBCT) for obtaining high quality features is proposed. In [4] a content based image retrieval method for diagnosis aid in medical fields is proposed. a model of wavelet coefficient distribution is used to enhance results, a weighted distance between signatures is used and an adapted wavelet base is proposed. In a similar approach to medical application in [5] an evaluation and comparison of the performance of four different and shape feature extraction methods for classification of benign and malignant micro calcifications in mammograms is proposed. clusters, and shape features were extracted for the DWT bands. For each set of features, most discriminating features and their optimal weights were found using real-valued and binary genetic algorithms (GA) utilizing a k-nearest-neighbor classifier.In [6] a new method for classification of human emotions based on multiwavelet transform of magnetic resonance imaging (MRI) images is proposed. The extracted features used as an input to multiclass least squares support vector machine (MC-LS-SVM) for classification of human emotions. In [7] a novel method towards multi-script identification at block level is proposed. The recognition is based upon features extracted using Discrete Cosine Transform (DCT) and Wavelets of Daubechies family. In [8] an Eyebrows identity authentication based on wavelet transform and support vector machine is proposed. The features of the eyebrows image are extracted by wavelet transform, and then classify them based on SVM. The system shows a low FAR of $0.22 \%$ and FRR $28 \%$. In [9] a new algorithm for image indexing and retrieval using Daubechies Wavelet Transform (DWT) is presented. In this DWT has been implemented by using wavelet sub-bands of R, G and B components of images from Coral-1000 database. An Adaptive wavelet-based image characterization is proposed. The wavelet basis was tuned to maximize the retrieval performance in a training dataset. The feasibility of using wavelet features and its advantages is observed from the literatures; also various mode of classification process is also developed. However in this process it is observed that the features are considered based on the extracted bands, however, these bands are not selective enough to define the actual property of the spectral bands, as these features are randomly scattered over the whole spectrum. These variations results in a non-uniform feature representation, which need to be normalized to uniform level representation so as the features are linearly represented. To achieve the objective of a linear feature representation in this work a new normalized feature representation for pattern recognition based in singular representation and its normalization for the wavelet spectral band is proposed.This paper 
proposed a new coding technique for pattern recognition based on the spectral features of subjects. In this approach, the spectral features such as power spectral density are used for pattern recognition. The rest of the paper is organized as follows: Section II gives the details of general pattern recognition system. The complete details of the proposed approach is given I section III. The experimental evaluation is carried out in section IV and finally the conclusions are provided in section $\mathrm{V}$.

\section{Pattern Recognition}

The image recovery systems are determined with generally the content features of the image named as shape or texture, color recognition. In this paper information of color-based is taken as the referencing index and using DWT method the spectral variation can be calculated. In input image information we can extract the frequency resolution information very efficiently using DWT method. Where the resolution information was passed as additional information for recovery in past, computational complexity increases because increasing the feature count result. To achieve the objective of image retrieval, the operation is performed in two stages, (i) training and (ii) testing. A Basic architecture for such a system is shown in figure 1.

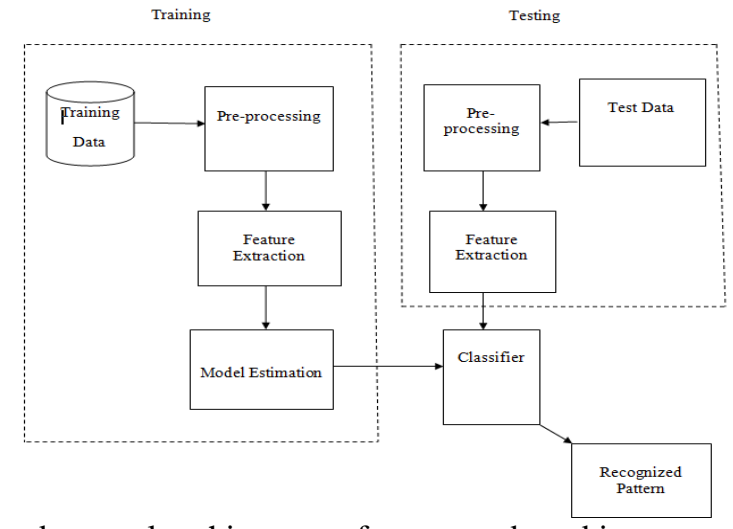

Figure 1: Fundamental architecture of a content based image retrieval system

In training and testing the samples are pre-processed for resizing, filtration and data precision. The preprocessing sample is further processes for feature extraction. In this stage image features are extracted namely shape or texture, color recognition. In this paper information of color-based is taken as the referencing index and using DWT method the spectral variation can be calculated. In input image information we can extract the frequency resolution information very efficiently using DWT method. Where the resolution information was passed as additional information for recovery in past, computational complexity increases because increasing the feature count result.

\section{Normalized Spectral Features For Pattern Recognition}

In a multi-resolution analysis, a scaling function $\phi(x)$ is employed to process the multi-resolution. The wavelet get decomposed into $a_{m, n}(f)$ called as approximate coefficients of $a_{m-1,1}$ and $c_{m, n}(f)$ termed as detail coefficients of a $\mathrm{m}_{-1,1}$ using a low-pass and a high-pass filter in cascade. The two-dimensional decomposition is carried out by the combination of two one-dimensional decomposition of wavelet transform. Two-dimensional discrete wavelet transform can be achieved by two 1-D DWT operations performing operations isolately on rows and columns. Firstly the row operation is performed to obtain two sub-bands by using 1-D DWT, one lowpass sub-band (L) and one high-pass sub-band $(\mathrm{H})$ as shown in Figure 4. The 1-D DWT image is transformed again to obtain four sub-bands by another 1-D DWT operation. Figure 4 shows the filter bank realization for the decomposition process of a 2-D DWT operation. The LL sub-band represents the approximate component of the image and other three sub-bands ( $\mathrm{LH}, \mathrm{HL}$ and $\mathrm{HH}$ ) represent the detail components. This is a non-uniform band splitting method that decomposes the lower frequency part into narrower bands and the high-pass output at each level termed as detail coefficients are left without any further decomposition. This procedure is done for all rows. Next, the filtering is done for each column of the intermediate data. The resulting two-dimensional array of coefficients contains four bands of data, each labeled as LL (low-low), HL (high-low), LH (low-high) and HH (high-high). A high wavelet coefficient (in absolute value) at a coarse resolution corresponds to a region with high global variations. The idea is to find a relevant point to represent this global variation by looking at wavelet coefficients at finer resolutions. A wavelet is an oscillating and attenuating function with zero integral. We study the image $f$ at the scales of $1 / 2,1 / 4, \ldots, 2^{j}, j \in \square$. the resolution decomposition of the color feature revels the color feature variation of the image. These spectral bands are processed to compute features using different feature descriptors such as the mean, standard deviation, entropy, energy etc. However all these 
features are extracted over the total extracted bands which are very random in distribution. Each of the variation in a distribution results in variation in the feature values. Hence in the process of obtaining a linear feature value a normalization process is proposed. The spectral bands of the wavelet filtration process have large spectral variations at a very nearby region. A spectral plot for a spectral band for a wavelet coefficient illustrates the variation, as shown in figure 2.

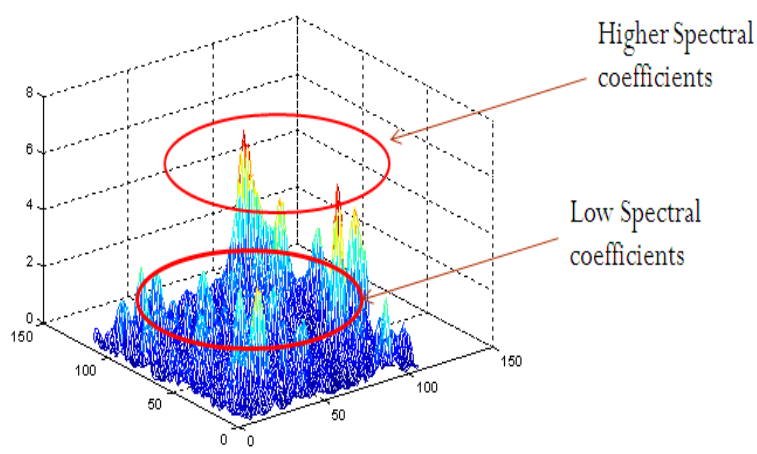

Figure 2. Spectral Band plot for a wavelet decomposed band

These spectral variations suppress the lower spectral coefficient and with it the information's content into the band is also suppressed. Hence these variations if normalized to a common scale could result in proper feature description. To develop the stated approach a normalization process over a singular feature is proposed. The proposed method is as outlined. For the obtained spectral bands a process of singular value decomposition (SVD) is carried out. This transformation transforms the obtained bands to eigen space which derive the variations in the obtained bands. In the process of SVD transformation, the band $\mathrm{B}_{\mathrm{i}}$ is decomposed into a singular matrix observation, where the variations are represented by a diagonal matrix. The SVD of an $N \times N$ band matrix has a decomposition form;

$\mathrm{F}=\mathrm{U} \times \mathrm{S} \times \mathrm{V}^{\mathrm{T}}(1)$

Where ' $\mathrm{U}$ ' and ' $\mathrm{V}$ ' are $\mathrm{N} \times \mathrm{N}$ orthogonal matrices and ' $\mathrm{S}$ ' is a $\mathrm{N} \times \mathrm{N}$ diagonal matrix here ' $\mathrm{S}$ ' diagonal elements represents the variation in the given coefficient. The variations for this coefficient are a non-linear distribution. This non-linearity is minimized by amplifying the ' $S$ ' matrix by a Norm parameter ' $\gamma$ '. To achieve the amplification a Norm to the normalized Singular matrix is made defined by;

$\mathrm{B}=\mathrm{U} \times \mathrm{S}^{\gamma} \mathrm{x} \mathrm{V}^{\mathrm{T}}(2)$

called the Normalized singular feature (NSF) representation. Where this Norm parameter ' $\gamma$ ' is varied in the range of $0 \leq \gamma \leq 1$. This range of value linearizes the ' $S$ ' matrix to one common level. The singular normalization when applied back to the band coefficient results in normalized band variations. The normalized bands are then process for feature descriptors. As the variations are linearizes to a common scale, the obtained features are linear in scale and any variation in the input observation will only effective to the feature rather to the band information. These features are then processed for classification, in the classification phase, the features are extracted from the test sample $\mathrm{x}$ using the proposed feature extraction algorithm, and then compared with the corresponding feature values of all the classes $\mathrm{k}$ stored in the feature library using the distance vector formula,

$D(M)=\sqrt{\sum_{j=0}^{N}[f(x)-f(M)]^{2}}$

where, $N$ is the number of features in $f_{j}(x)$, where $j$ represents the $j^{\text {th }}$ feature of the test sample $x$, while $f$ $j(M)$ represents the $j^{\text {th }}$ feature of $M^{\text {th }}$ class in the library. The test is classified using the K-nearest neighbors (K$\mathrm{NN}$ ) classifier. In the K-NN classifier, the class of the test sample is decided by the majority class among the K nearest neighbors. A neighbor is deemed nearest if it has the smallest distance in the feature space. In order to avoid a tied vote, it is preferable to choose $\mathrm{K}$ to be an odd number. The experiments are performed choosing $\mathrm{K}=3$. The classification of the feature vector is performed based on the Euclidian distance approach. For a given test image $\operatorname{Tr} \sim \epsilon \mathrm{R}^{\mathrm{m} \times \mathrm{n}}$ is transformed into a feature matrix $\mathrm{Yr} \in \mathrm{R}^{\mathrm{r} \times \mathrm{c}}$. For the computed feature the distance between a test image $\mathrm{T}$ and a traning images $\mathrm{X}_{\mathrm{i}}^{(\mathrm{j})}$ is calculated by $\mathrm{R}_{\mathrm{ji}}=\delta\left(\mathrm{Y}, \mathrm{X}_{\mathrm{i}}^{(\mathrm{j})}\right)=\left|Y-X_{i}^{(j)}\right|$, using a Frobenius norm. From the Retrieve top 8 subjects of the database according to the rank of $\mathrm{R}_{\mathrm{ji}}$ given by arg Rank ${ }_{j}\left\{R_{j i}=\delta\left(Y, X_{i}^{(j)}\right), 1 \leq i \leq N_{j}\right\}$. The image with the highest Rank is declared as the recognized image. For the evaluation of the image retrieval a performance analysis is carried out for spatial similar images and compared with the conventional retrieval system. 


\section{Results And Discussion}

To evaluate the process of proposed normalized feature descriptor logic, a test analysis is carried out over different orientation test samples. These test samples were processed from Brainweb [16] data set. The data set is formed with various orientations of a test sample. Few samples of this data set are as shown in figure 3.

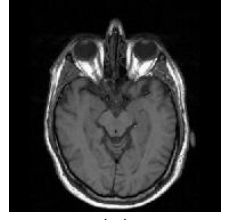

(a)

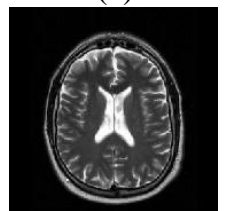

(d)

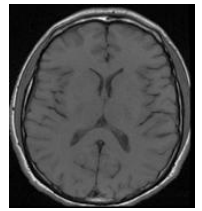

$(\mathrm{g})$

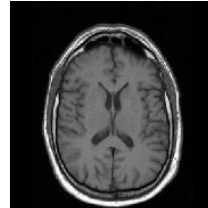

(b)

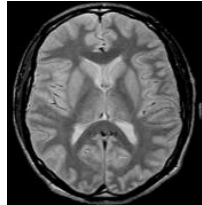

(e)

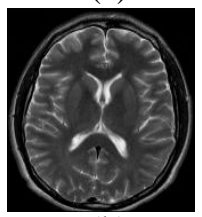

(h)

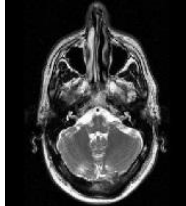

(c)

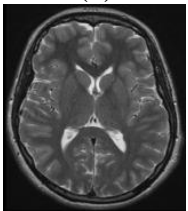

(f)

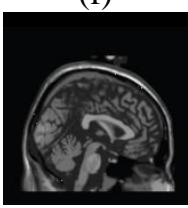

(i)

Figure 3: Database samples from MRI image Dataset.

To evaluate the process of normalized feature processing a test sample with different orientation is passed to the developed system. The obtained observations for the developed system is as illustrated below,

\section{Sample I: Sample with $0^{0}$ orientation}

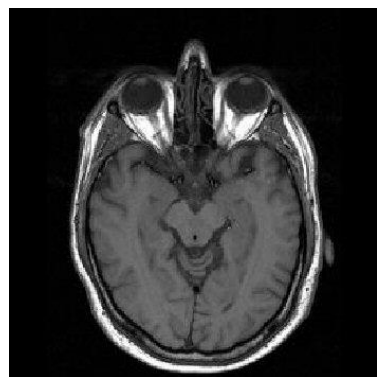

Figure 4: Original Query sample at 0 orientation

A selected test sample for the recognition process is shown in figure 4.

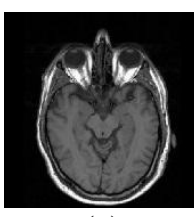

(a)

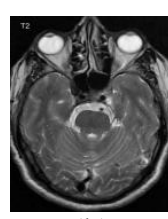

(b)

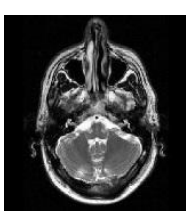

(c)

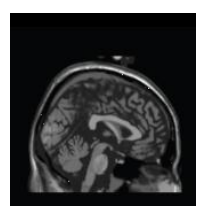

(d)

Figure 5: Top 4 classified sample using Normalized-Singular Feature Descriptor

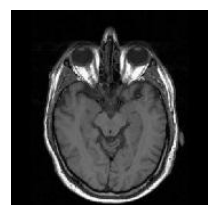

(a)

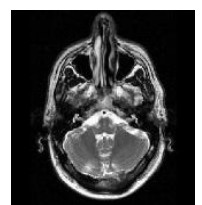

(b)

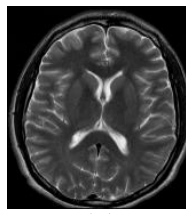

(c)

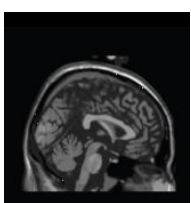

(d)

Figure 6: Top 4 classified sample using Wavelet Feature Descriptor 
The top 4 classified sample from the trained data base for the proposed NSF and Wavelet feature descriptor is shown in figure 5 and 6 respectively.

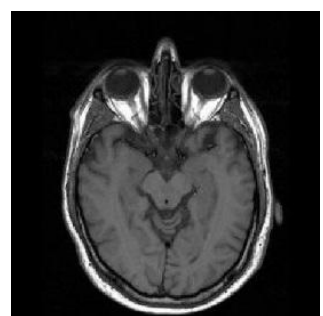

(a)

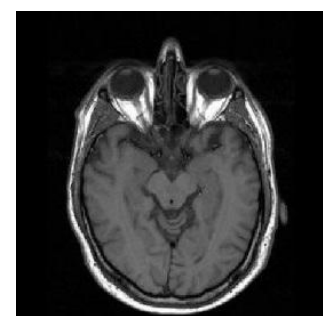

(b)

Figure 7: Final retrieved sample (a) Using NSF (b) Wavelet Feature Descriptor

The top retrieved sample from the data base after classification for the two methods is shown in figure 7 (a) and (b) respectively. This test sample is then evaluated over different orientation and the obtained results are as illustrated below;

\section{Sample II: Sample with $15^{0}$ orientation}

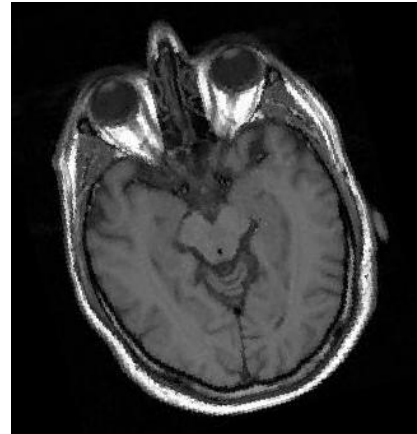

Figure 8: Query sample at orientation of $15^{0}$

The test sample is oriented by $15^{\circ}$ orientation, and passed as a query sample to the developed system. The features are extracted using conventional wavelet bands features and normalized feature bands. Using theses feature bands, recognition process is carried out using K-NN classifier. The obtained results of the classified observations are illustrated in figure 9.

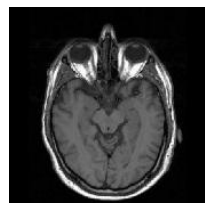

(a)

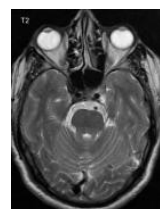

(b)

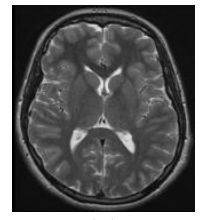

(c)

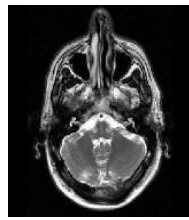

(d)

Figure 9: Top 4 classified sample using Normalized-Singular Feature Descriptor

When applied with orientation it is observed that the features are extracted from the normalized bands hence the feature variation due to spectral orientation is not effective, as these bands are normalized to a uniform scale. However this variation is retained in wavelet based feature descriptor hence the classification process is affected.

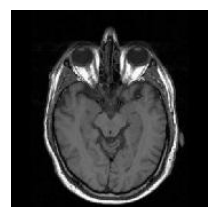

(a)

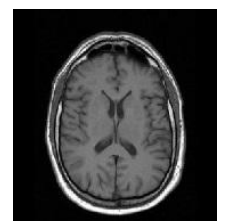

(b)

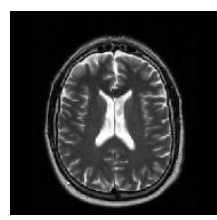

(c)

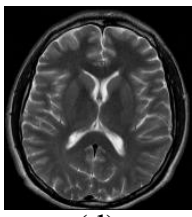

(d)

Figure 10: Top 4 classified sample using Wavelet Feature Descriptor 


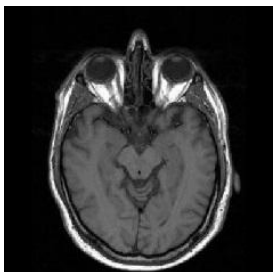

(a)

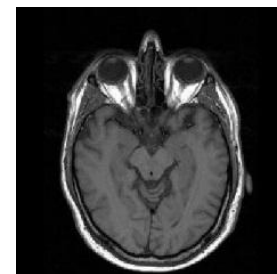

(b)

Figure 11: Final retrieved sample (a) Using NSF (b) Wavelet Feature Descriptor Sample III: Sample with $4^{\circ}$ orientation

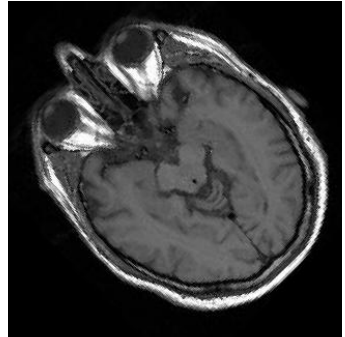

Figure 12: Query sample at $45^{\circ}$ orientation

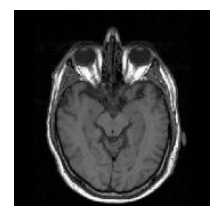

(a)

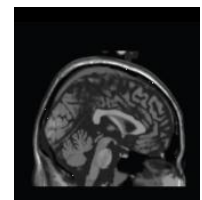

(b)

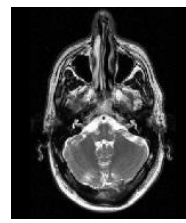

(c)

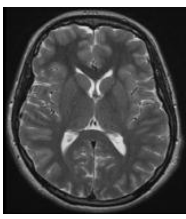

(d)

Figure 13: Top 4 classified sample using Normalized-Singular Feature Descriptor

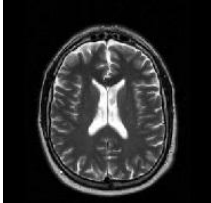

(a)

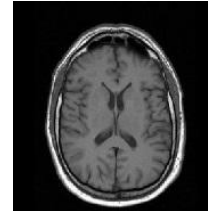

(b)

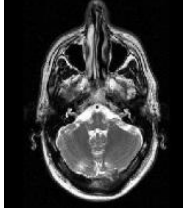

(c)

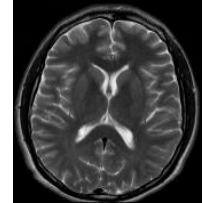

(d)

Figure 14: Top 4 classified sample using Normalized-Singular Feature Descriptor

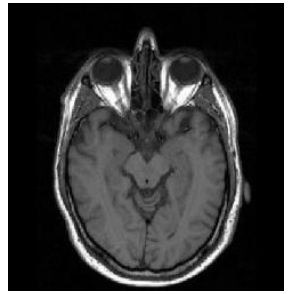

(a)

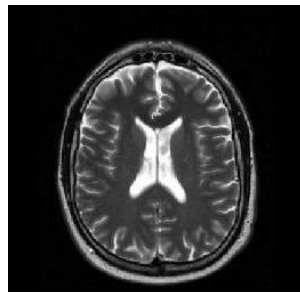

(b)

Figure 15: Final retrieved sample (a) Using NSF (b) Wavelet Feature Descriptor

An analysis is carried out for different test samples at different orientations. The test result for these samples is outlined in figure 16.

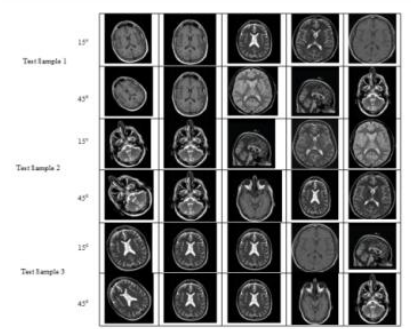

Figure 16: observations obtained for different test samples at $15,45^{\circ}$ orientations 


\section{Conclusion}

The multi spectral band selection approach, based on power spectral density, gives a higher performance in retrieval as in comparison to the liner modeling of multi-wavelet feature representation. In the coding approach to feature selection, the dominant band selection results in more informative feature selection process, which leads to higher retrieval accuracy. The optimal band selection criterion suggested, derives the most domain bands in the decomposed feature bands, this selection approach extract the highest informative regions of the test sample, which intern results in higher retrieval performance.

\section{References}

[1]. Te-Wei Chiang, Tien-Wei Tsai, "Content-Based Image Retrieval via the Multiresolution Wavelet Features of Interest", Journal of Information Technology and Applications Vol. 1 No. 3, December, 2006.

[2]. Vinayak D. Shinde, Vijay M. Mane, “ Pattern Recognition using Multilevel Wavelet Transform”, International Journal of Computer Applications, Volume 49- No.2, July 2012

[3]. ZhongliangLuo, "Iris Feature Extraction and Recognition Based on Wavelet-Based Contourlet Transform", Procedia Engineering, Elsevier, 2012.

[4]. Mathieu Lamard, Guy Cazuguel, Gw enoleQuellec, Lynda Bekri, Christian Roux, "Content Based Image Retrieval based on Wavelet Transform coefficients distribution", Conference proceedings : Annual International Conference of the IEEE Engineering in Medicine and Biology Society., IEEE, 2007.

[5]. Hamid Soltanian-Zadeha, FarshidRafiee-Rad, SiamakPourabdollah-Nejad D, "Comparison of multiwavelet, wavelet, Haralick, and shape features for micro calcification classification in mammograms", journal of Pattern Recognition, Pergamon, Elsevier, 2004.

[6]. Varun Bajaj, Ram BilasPachori, "Classification of human emotions based on multiwavelet transform of EEG signals", AASRI Procedia, Elsevier, 2012

[7]. Jomy John, Pramod K.V., KannanBalakrishnan, "Unconstrained Handwritten Malayalam Character Recognition using Wavelet Transform and Support vector Machine Classifier", Procedia Engineering, Elsevier, 2012.

[8]. Cao Jun-bin, Yang Haitao, Ding Lili, "Eyebrows Identity Authentication Based on Wavelet Transform and Support Vector Machine", Physics Procedia, Elsevier, 2012.

[9]. SheetalJagannathDalavi, Mahadev S. Patil ,Sanjay R. Patil, "Content Based Image Retrieval by Using Daubechies Wavelet Transform”, International Conference on Innovations in Engineering and Technology (ICIET’14), IEEE, 2014. 